\title{
EDITORIAL
}

\section{Identification of AF Drivers: Toward a Mechanistic Basis of AF Ablation}

\author{
KENTARO YOSHIDA, M.D. and AMAN CHUGH, M.D. \\ From the Division of Cardiovascular Medicine, University of Michigan, Ann Arbor, Michigan
}

While the ablation endpoint in patients with paroxysmal atrial fibrillation (AF) has been largely standardized, the optimal approach for patients with persistent AF remains unknown. Among the latter, it is clear that pulmonary vein (PV) isolation alone is insufficient. ${ }^{1}$ In an effort to improve outcomes in patients with persistent AF, left atrial (LA) substrate modification has been shown to be effective. ${ }^{2,3}$ This may be accomplished by linear ablation and/or ablation of complex, fractionated electrograms (CFAEs). Although CFAEs may be markers of sites that are critical to the fibrillatory process, i.e., drivers, it is also possible that they represent passive phenomena as a result of wave collision, tissue anisotropy, and slow conduction. ${ }^{4}$ Even if one uses a relatively stringent definition of a CFAE site, e.g., continuous electrical activity, the likelihood that ablation at such a site will have a favorable impact on the AF cycle length is modest. ${ }^{5}$ The fact that such sites may be found away from the PV region in patients with paroxysmal AF also suggests that they may be nonspecific. ${ }^{6}$ Also, a randomized study failed to show an incremental benefit of CFAE ablation in patients with persistent AF. ${ }^{7}$ Thus, it is possible that CFAE ablation results in more tissue destruction than perhaps necessary to eliminate AF. This has important implications with regards to procedure times, complication rates, and atrial transport function.

\section{Prior Studies Using Multielectrode Catheters}

Clinical studies involving electrogram-guided ablation typically used an ablation catheter for mapping purposes. ${ }^{8}$ Obviously, the inability to map adjacent areas simultaneously makes it impossible to distinguish between active and passive phenomena. In an attempt to overcome this limitation, several investigators have utilized multielectrode catheters capable of recording over a larger surface area of the left atrium. Rostock et al. ${ }^{9}$ investigated CFAE sites in patients with paroxysmal AF using a 20-pole PentaRay catheter (Biosense Webster, Diamond Bar, CA, USA) and reported that in

\footnotetext{
Address for reprints: Aman Chugh, M.D., Cardiovascular Center, SPC 5853, 1500 E. Medical Center Dr., Ann Arbor, MI 48109-5853. Fax: 734-936-7026; e-mail: achugh@umich.edu

the majority of cases, there was nearly simultaneous activation of the mapping field covering only a limited part of the AF cycle length. This pattern suggests passive activation from an AF driving process beyond the mapped area and thus underscores the limited specificity of CFAE sites. In another study using the same catheter, Takahashi and colleagues performed high-density mapping during AF. ${ }^{10}$ They noted spontaneous focal activity, indicated by centrifugal activation from the center of the multipolar catheter, from 13 sites (from 12 of 24 study patients) in the left atrium during ongoing AF. Notably, AF terminated without ablation at these sites in all patients, although two patients did require additional radiofrequency energy delivery at these sites for arrhythmia recurrence. The authors concluded that areas responsible for the focal activity may represent "secondary drivers" since AF termination did not require their elimination. Although mapping with multipolar catheters further improved our understanding of mechanisms of AF, it is still insufficient to guide ablation of persistent AF in most patients.

\section{The Driver Hypothesis}

Several investigators have very elegantly demonstrated the presence of AF "drivers" in animal models. These drivers manifest not only the fastest activation rates at a point in time, but also radial decay in rate and organization away from the source (fibrillatory conduction). ${ }^{11-14}$ The ability to identify these areas in patients may greatly simplify catheter ablation in patients with persistent AF. However, although the driver hypothesis has been demonstrated in animal studies, its evidence in human AF is limited. ${ }^{15-17}$ One of the reasons for this discrepancy may have to do with the fact that the spatial resolution of optical mapping in animal studies is much higher $(<0.5 \mathrm{~mm})$, as compared with mapping with tools currently available in clinical electrophysiology. ${ }^{12}$

In this issue of the Journal, Krummen et al. strive to overcome this limitation and investigated gradients of rate and organization during AF in 24 patients undergoing catheter ablation. The investigators performed LA mapping with a basket catheter, which allows for simultaneous sampling of a broad area of the left atrium and with higher resolution as compared to other

(C)2009, The Authors. Journal compilation (C)2009 Wiley Periodicals, Inc. 
multipolar catheters. In keeping with the findings from seminal studies in animals, the authors used a very rigorous definition of a driver. In order for a source to be classified as a driver, it must not only exhibit the fastest rate at a particular point in time, but also radial decrement in rate and organization.

The authors found potential driver sources in approximately $50 \%$ of patients with paroxysmal and persistent AF. These sites demonstrated a higher activation frequency and regularity than adjacent and distant sites, consistent with a centrifugal gradient in rate and organization. As expected, in patients with paroxysmal AF, the sites were localized to the PV region, and in persistent $\mathrm{AF}$, to non-PV sites, such as coronary sinus, roof of the left atrium, and the mitral annulus. Radiofrequency energy delivery at the driver sites led to slowing of the AF cycle length in three patients and AF termination in two patients. Conversely, when these driver sites were not ablated, the $\mathrm{AF}$ cycle length failed to prolong and was unchanged at the end of the procedure. The small sample size precludes a meaningful comparison of outcomes among patients in whom ablation did or did not traverse a driver site.

Although gradients of $\mathrm{AF}$ frequency have been documented previously,,$^{5,17,18}$ the frequency maps in these studies were constructed by sequential point-by-point mapping. Since drivers may drift over time, ${ }^{19}$ a major advantage of this study is that data were gleaned from simultaneous mapping of a large part of the left atrium. In fact, the authors noted that simulated sequential mapping was less effective in identifying a potential driver than simultaneous mapping.

\section{Mapping of Drivers during Ablation of Persistent AF?}

Identification of driver sites prior to ablation is intuitive and has the potential for making the ab-

\section{References}

1. Oral H, Knight BP, Tada H, Ozaydin M, Chugh A, Hassan S, Scharf $\mathrm{C}$, et al. Pulmonary vein isolation for paroxysmal and persistent atrial fibrillation. Circulation 2002; 105:1077-1081.

2. O’Neill MD, Wright M, Knecht S, Jais P, Hocini M, Takahashi Y, Jonsson A, et al. Long-term follow-up of persistent atrial fibrillation ablation using termination as a procedural endpoint. Eur Heart J 2009; 30:1105-1112.

3. Haissaguerre M, Hocini M, Sanders P, Sacher F, Rotter M, Takahashi Y, Rostock T, et al. Catheter ablation of long-lasting persistent atrial fibrillation: Clinical outcome and mechanisms of subsequent arrhythmias. J Cardiovasc Electrophysiol 2005; 16:11381147

4. Konings KT, Smeets JL, Penn OC, Wellens HJ, Allessie MA. Configuration of unipolar atrial electrograms during electrically induced atrial fibrillation in humans. Circulation 1997; 95:1231-1241.

5. Takahashi Y, O’Neill MD, Hocini M, Dubois R, Matsuo S, Knecht S, Mahapatra S, et al. Characterization of electrograms associated with termination of chronic atrial fibrillation by catheter ablation. J Am Coll Cardiol 2008; 51:1003-1010. lation procedure more efficient. However, several limitations must be overcome before "driver mapping" can be adopted as a clinical tool during ablation of persistent AF. First, it must be noted that frequency analysis in this study was performed off-line. In order to use frequency data to guide ablation, signal processing should be able to be performed real-time and efficiently. Second, simultaneous LA mapping requires insertion of a basket catheter that is not routinely used during ablation procedures for AF. Since patients with persistent AF may harbor more than one driver, ${ }^{20}$ the time needed for redeployment and remapping is a disadvantage. The risk of thrombo-embolism should also be considered. ${ }^{21}$ The definition of "driver" may need to be modified since only $50 \%$ of patients were found to harbor drivers in the current study. Most importantly, the results need to be validated in larger, preferably randomized study to determine whether AF ablation guided by identification of drivers has incremental benefit as compared to CFAE ablation.

The past decade has witnessed remarkable progress in catheter ablation of AF. In this short period of time, the procedure has evolved dramatically, so much so that it is now possible to eliminate the arrhythmia even in those with longlasting $\mathrm{AF}$ and structural heart disease. However, our understanding of the basic mechanisms of AF has not kept pace with these advances. Krummen et al. have extended this knowledge base by showing that it is possible to identify sites critical to the fibrillatory process based on rate and organization. The logical extension of these findings is that ideally, such sites can be identified a priori and then can be efficiently eliminated without ablating noncritical sites. Hopefully, in the very near future, better tools will become available, capable of readily identifying driver sites real-time, ushering in the era of mechanistic rather than empiric $\mathrm{AF}$ ablation.

6. Tada H, Yoshida K, Chugh A, Boonyapisit W, Crawford T, Sarrazin JF, Kuhne M, et al. Prevalence and characteristics of continuous electrical activity in patients with paroxysmal and persistent atrial fibrillation. J Cardiovasc Electrophysiol 2008; 19:606-612.

7. Oral H, Chugh A, Yoshida K, Sarrazin JF, Kuhne M, Crawford T, Chalfoun N, et al. A randomized assessment of the incremental role of ablation of complex fractionated atrial electrograms after antral pulmonary vein isolation for long-lasting persistent atrial fibrillation. J Am Coll Cardiol 2009; 53:782-789.

8. Nademanee K, McKenzie J, Kosar E, Schwab M, Sunsaneewitayakul B, Vasavakul T, Khunnawat C, et al. A new approach for catheter ablation of atrial fibrillation: Mapping of the electrophysiologic substrate. J Am Coll Cardiol 2004; 43:2044-2053.

9. Rostock T, Rotter M, Sanders P, Takahashi Y, Jais P, Hocini M, Hsu LF, et al. High-density activation mapping of fractionated electrograms in the atria of patients with paroxysmal atrial fibrillation. Heart Rhythm 2006; 3:27-34.

10. Takahashi Y, Hocini M, O’Neill MD, Sanders P, Rotter M, Rostock $\mathrm{T}$, Jonsson A, et al. Sites of focal atrial activity characterized by 


\section{MAPPING OF AF DRIVERS}

endocardial mapping during atrial fibrillation. J Am Coll Cardiol 2006; 47:2005-2012.

11. Berenfeld O, Zaitsev AV, Mironov SF, Pertsov AM, Jalife J. Frequency-dependent breakdown of wave propagation into fibrillatory conduction across the pectinate muscle network in the isolated sheep right atrium. Circ Res 2002; 90:1173-1180.

12. Kalifa J, Tanaka K, Zaitsev AV, Warren M, Vaidyanathan R, Auerbach D, Pandit S, et al. Mechanisms of wave fractionation at boundaries of high-frequency excitation in the posterior left atrium of the isolated sheep heart during atrial fibrillation. Circulation 2006; 113:626-633.

13. Mandapati R, Skanes A, Chen J, Berenfeld O, Jalife J. Stable microreentrant sources as a mechanism of atrial fibrillation in the isolated sheep heart. Circulation 2000; 101:194-199.

14. Berenfeld O, Mandapati R, Dixit S, Skanes AC, Chen J, Mansour M, Jalife J. Spatially distributed dominant excitation frequencies reveal hidden organization in atrial fibrillation in the Langendorffperfused sheep heart. J Cardiovasc Electrophysiol 2000; 11:869879.

15. Lazar S, Dixit S, Marchlinski FE, Callans DJ, Gerstenfeld EP. Presence of left-to-right atrial frequency gradient in paroxysmal but not persistent atrial fibrillation in humans. Circulation 2004; 110:31813186.
16. Sahadevan J, Ryu K, Peltz L, Khrestian CM, Stewart RW, Markowitz AH, Waldo AL. Epicardial mapping of chronic atrial fibrillation in patients: Preliminary observations. Circulation 2004; 110:32933299 .

17. Sanders P, Berenfeld O, Hocini M, Jais P, Vaidyanathan R, Hsu LF, Garrigue S, et al. Spectral analysis identifies sites of high-frequency activity maintaining atrial fibrillation in humans. Circulation 2005; 112:789-797.

18. Takahashi Y, Jais P, Hocini M, Sanders P, Rotter M, Rostock T, Hsu $\mathrm{LF}$, et al. Shortening of fibrillatory cycle length in the pulmonary vein during vagal excitation. J Am Coll Cardiol 2006; 47:774-780.

19. Zlochiver S, Yamazaki M, Kalifa J, Berenfeld O. Rotor meandering contributes to irregularity in electrograms during atrial fibrillation. Heart Rhythm 2008; 5:846-854.

20. Yoshida K, Chugh A, Ulfarsson M, Good E, Kuhne M, Crawford T, Sarrazin JF, et al. Relationship between the spectral characteristics of atrial fibrillation and atrial tachycardias that occur after catheter ablation of atrial fibrillation. Heart Rhythm 2009; 6:11-17.

21. Arentz T, Von Rosenthal J, Weber R, Burkle G, Blum T, Stockinger J, Neumann FJ, et al. Effects of circumferential ostial radiofrequency lesions on pulmonary vein activation recorded with a multipolar basket catheter. J Cardiovasc Electrophysiol 2005; 16:302308. 